\title{
Global Incidence, Progression, and Risk Factors of Age-Related Macular Degeneration and Projection of Disease Statistics in 30 Years: A Modeling Study
}

\author{
Yuye Wang $^{\mathrm{a}}$ Yifan Zhong $^{\mathrm{b}}$ Liang Zhang ${ }^{\mathrm{c}}$ Qijun Wu ${ }^{\mathrm{d}}$ Yihchung Tham $^{\mathrm{e}}$ \\ Tyler Hyungtaek Rim ${ }^{\mathrm{e}}$ David Mutugi Kithinji ${ }^{\mathrm{f}} \quad$ Jingyang Wu ${ }^{\mathrm{b}}$ Chingyu Cheng ${ }^{\mathrm{c}, \mathrm{e}}$

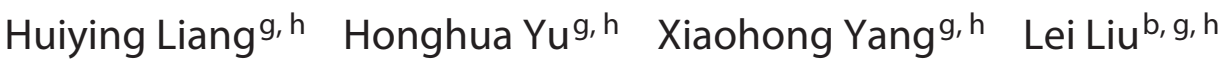 \\ aDepartment of Neurology, The First affiliated Hospital of China Medical University, Shenyang, China; ${ }^{b}$ Department of \\ Ophthalmology, The First affiliated Hospital of China Medical University, Shenyang, China; 'Singapore Eye Research \\ Institute, Singapore National Eye Centre, Singapore City, Singapore; ${ }^{d}$ Department of Clinical Epidemiology, Shengjing \\ Hospital of China Medical University, Shenyang, China; ${ }^{\circ}$ Ophthalmology \& Visual Sciences Academic Clinical Program \\ (Eye ACP), Duke-NUS Medical School, Singapore City, Singapore; fInternational Education School, China Medical \\ University, Shenyang, China; ${ }^{9}$ Department of Ophthalmology, Guangdong Eye Institute, Guangdong Provincial \\ People's Hospital, Guangdong Academy of Medical Sciences, Guangzhou, China; hSchool of Medicine, South China \\ University of Technology, Guangzhou, China
}

\section{Keywords}

Age-related macular degeneration · Incidence · Progression · Risk factors

\footnotetext{
Abstract

Objective: Age-related macular degeneration(AMD) has become a major cause of visual impairment worldwide, especially in the elderly. Estimates of incidence, progression rates, and risk factors of AMD vary among studies, complicating the understanding of its epidemiology. Methods: For this systematic review and meta-analysis, literature published up to March 1, 2021, was searched in both English and Chinese databases. Hierarchical Bayesian approaches were used to estimate pooled incidence, progression, and 95\% credible intervals (Crls). Results: Thirty studies were included. The pooled annual early and late AMD incidence rates were 1.59 (95\% Crl: 1.18-2.11) and 0.23 (95\% Crl: 0.14-0.34) per 100 person-years, respectively. The annual progression
}

karger@karger.com www.karger.com/ger

Karger $\stackrel{\text { ' }}{5}$

GOPEN ACCESS
(C) 2021 The Author(s)

Published by S. Karger AG, Basel

This is an Open Access article licensed under the Creative Commons Attribution-NonCommercial-4.0 International License (CC BY-NC) (http://www.karger.com/Services/OpenAccessLicense), applicable to the online version of the article only. Usage and distribution for commercial purposes requires written permission. rate of AMD was 5.5 (95\% Crl: 2.3-8.8) per 100 person-years. Smoking was an independent risk factor for both early and late AMD, whereas age, high-density lipoprotein cholesterol, and alcohol consumption were risk factors for early AMD incidence only. The projected number of new cases of early and late AMD in 2050 would be 39.05 million (95\% Crl: 23.1263.57) and 6.41 million (95\% Crl: 3.37-13.22), respectively. Conclusion: The prediction the number of new cases of AMD is not equal across the globe. Our findings indicate the need for more rigorous control and prevention measures in AMD focus on its risk factors for early intervention. The epidemiological estimates reported in this study could inform to identify effective strategies for preventing AMD worldwide.

(C) 2021 The Author(s)

Published by S. Karger AG, Basel

Yuye Wang, Yifan Zhong and Liang Zhang contribute equally to this work. 


\section{Introduction}

Age-related macular degeneration (AMD) has become the most common cause of blindness among people over 60 years old, particularly in developed countries $[1,2]$. As the population of older persons in the world is expected to double by 2050, it is important to examine how AMD occurs over time and what contributes to its development to point the way for related health services and protocol. Previously, there were several meta-analyses focused on the prevalence of AMD [3-6], which measures the proportion of the disease in the population. However, incidence is a key measure to examine new cases over time, allowing for forecasting of current demands for related health care services. Additionally, progression is an important indicator of the stage and severity of the disease. In particular, there is no systematic review of both incidence and progression of AMD despite multiple individual studies. Interpreting incidence data from such different studies is challenging because of wide variation in estimates between countries and regions, due to differences in study methods, diagnosis criteria, and time trends. Generally, according to the grading protocol of the Wisconsin Age-Related Macular Degeneration Grading System (WAMDGS) [7] and international classification and grading system (IGS) [8], early AMD can be defined by the presence of signs of any size drusen and pigmentary abnormalities, or by the presence of a large size drusen over $125 \mu \mathrm{m}$ in diameter but without signs of late AMD. Late AMD can be defined as follows: geographic atrophy of the retinal pigment epithelium or pigment epithelial detachment, subretinal hemorrhage or subretinal new vessel, or subretinal scar or photocoagulation treatment scar. There is little summarized data on AMD incidence or progression to guide global strategies for disease management. A prior meta-analysis reported that annual incidence of late AMD in white Americans was 3.5 per 1,000 aged $>50$ years, equivalent to 293,000 new cases in white Americans per year [9]. However, there is a lack of robust estimates of the worldwide incidence and progression of both early and late AMD in the general population.

Besides, in order to better understand of etiology and provide pathways to AMD prevention, it is necessary to analyze the risk factors. Risk factors can be divided into demographic factors, such as age, sex and ethnicity, and lifestyle factors, such as smoking and alcohol consumption [10]. However, numerous risk factors on AMD have been reported but the evidence and strength of association varies. Currently, little is known about the risk factors of AMD based on longitudinal cohort studies providing strong evi- dence worldwide, which are very important for clinical or public healthy staff to intervene and prevent as soon as possible and reduce the incidence of ophthalmological caries. Generally, robust data on incidence, progression and risk factors of both early and late AMD and their burden are important for development of major public health strategies, such as screening and prevention programs.

\section{Methods}

This systematic review was conducted and reported in accordance with the Reporting Checklist for Meta-analyses of Observational Studies (MOOSE). The completed MOOSE checklist is available in the online suppl. material (for all online suppl. material, see www.karger.com/doi/10.1159/000518822). Further, this study is registered with PROSPERO, number CRD42019118832.

\section{Search Strategy and Selection Criteria}

For this systematic review and meta-analysis, 2 authors (Y.W. and L.L.) independently searched PubMed, Web of Science, EMBASE, China National Knowledge Infrastructure (CNKI), Wanfang, and ViP databases for relevant articles published up to March 1,2021. Detailed search terms (formatted for PubMed search): ["Macular Degeneration" [Mesh] AND ("Incidence" [Mesh] OR "Epidemiology" [Mesh] OR "Longitudinal Studies” [Mesh] OR “Cohort Studies" [Mesh])\}; \{(“age-related maculopathy” [All Fields] OR "age-related maculopathy" [All Fields] OR "age-related macular degeneration" [All Fields] OR "age-related macular degeneration" [All Fields] OR "macular degeneration" [All Fields]) AND ("incidence" [All Fields] OR “progression” [All Fields] OR “epidemiology” [All Fields] OR "risk factors" [All Fields])\}, without language restrictions. Additionally, reference lists of identified articles were also searched to identify other relevant literatures. Two reviewers (Y.W. and Y.Z.) evaluated all included studies independently. Disagreements were discussed with another investigator (L.L.) and resolved by consensus.

Inclusion criteria were: (1) longitudinal or cohort studies on incidence or progression of AMD diagnosed by retinal photographs and standardized grading classifications (WAMDGS [7], IGS [8], or the Barbados Eye Studies Grading Protocol [BISEDGP] [11]), (2) AMD reported as an individual entity, and (3) the data included crude figures or enabled crude calculation of AMD incidence or progression. Studies with a greater amount of person-years or more sophisticated case-finding methods that reported on similar study populations but not conducted during the same time periods were also included. There were no restrictions regarding publication year. Exclusion criteria were as follows: (1) duplicated articles; (2) reports not written in English or Chinese; (3) reports number of eyes with AMD as opposed to number of individuals; (4) dynamic population; and (5) not population based or longitudinal cohort study.

\section{Data Extraction and Quality Assessment}

A data extraction spreadsheet was used to collect information on the study and participant characteristics from each of the included studies. Relevant information was extracted from the included studies by 2 researchers (Y.W. and L.L.). Publication authors were contacted for missing data on incidence or progression of AMD when necessary. We assessed the quality of included stud- 
Fig. 1. Flowchart: study identification and selection. AMD, age-related macular degeneration.

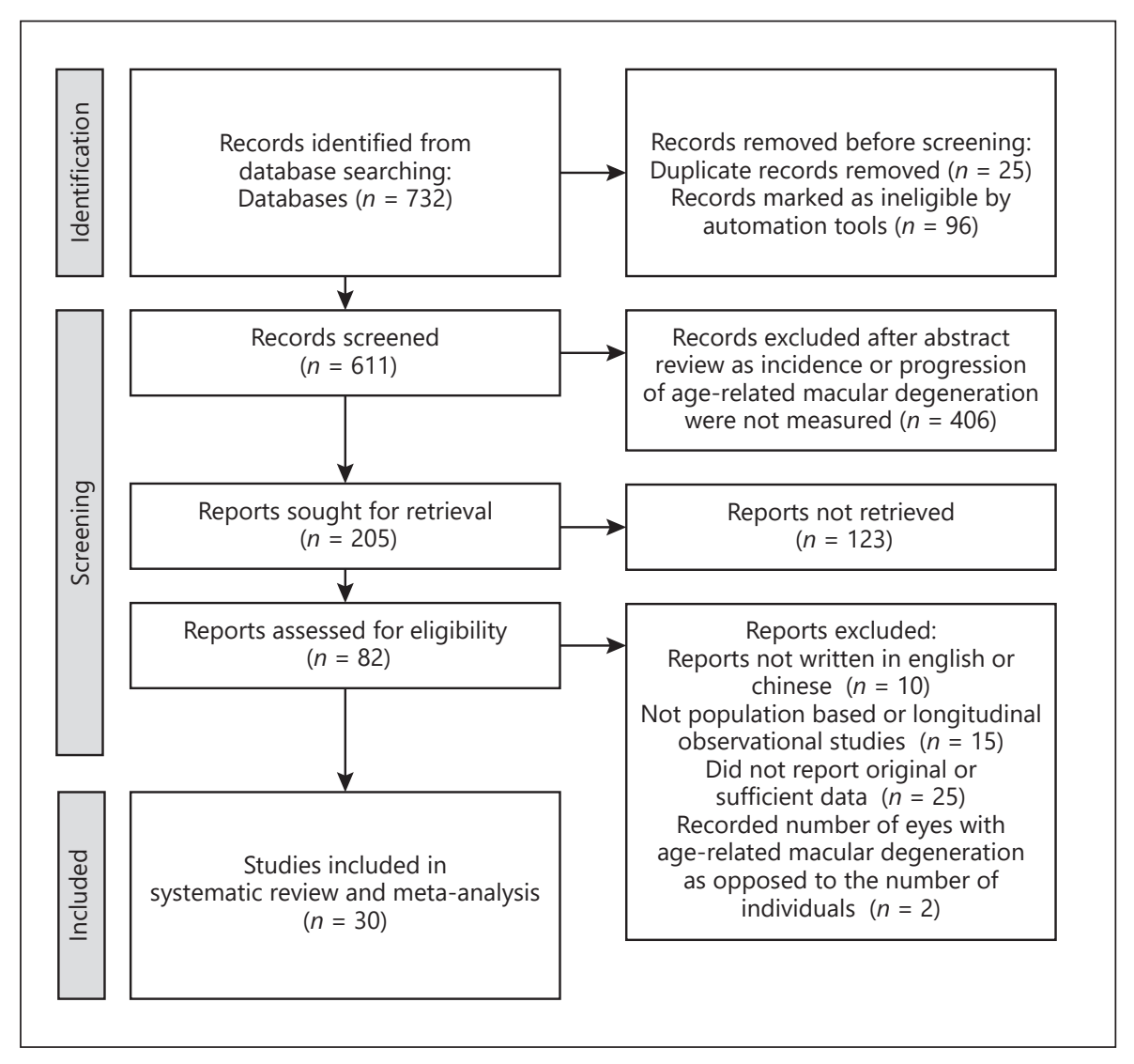

ies using an assessment scale based on the Newcastle-Ottawa Scale (NOS, details in online suppl. Method) [12, 13]. From this, studies were judged to be at low risk of bias ( $\geq 3$ points) or high risk of bias $(<3$ points). Data were double-checked and reviewed by a statistician (L.Z.). In the event of any disagreement on extracted data, the article was assessed again and discussed between the 3 reviewers (Y.W., Y.Z., and L.L.) until an agreement was achieved.

Incidence of AMD was defined as the development of early AMD or late AMD [14]. Progression of AMD was defined as development of advanced AMD in one or both eyes with early AMD at baseline of the study [15].

\section{Statistical Analysis}

Hierarchical Bayesian approach was used to estimate the pooled AMD annual incidence of the population along with $95 \%$ credible intervals (CrIs). Projections of AMD were estimated based on the World Population Prospects of the United Nations [16]. Age group-specific annual incidence rates were assumed to be constant over the next 30 years for our global projection for the year 2050, since Bayesian hypothesis testing of the study baseline year covariate (Bayes factor [BF] is 0.17 for early AMD and 0.19 for late $\mathrm{AMD}$ ) in our review showed no trend for incidence from year 1987 to 2010, whereas BF describes level of statistical evidence: negative evidence $(\mathrm{BF}<1)$, weak $(\mathrm{BF} 1-3)$, substantial (BF $3-10)$, strong (BF 10-30), very strong (BF 30-100), and decisive $(\mathrm{BF}>100)$ [17]. Bayesian meta-regression models were generated to assess the association between incidence of AMD and relevant factors. Since studies varied in length of follow-up, annual inci- dence was calculated based on exponential assumptions and the formula used by a previous systematic review [9]: $-\ln (1-S) / t$, where $\ln ()$ is the natural $\operatorname{logarithm}$ function, $S$ is the cumulative incidence over years and $t$ is the duration of years of follow-up.

In order to investigate the demographic, lifestyle, and healthrelated factors related to AMD incidence, Hierarchical Bayesian approach was used to calculate the pooled effect estimates. Since the incidences of both early and late AMD were very low $(<10 \%)$, the 3 measures (hazard ratio, relative risk, and odds ratio [OR]) of association were expected to yield similar estimates of OR [18]. Therefore, we put together all the OR estimates as appropriately as possible to ensure the comprehensiveness of the analysis and maximize the statistical power $[18,19]$. Forest plots are used to display statistical analysis results.

Standard $\chi^{2}$ test with $I^{2}$ statistics (the percentage of variability in incidence estimation due to heterogeneity rather than sampling error or chance) and $p$ values were used to assess between-study heterogeneity. Funnel plot and Egger test for funnel plot asymmetry were used to assess study publication bias.

\section{Results}

\section{Characteristics of Included Studies regarding}

As shown in Figure 1, the initial literature search returned 732 citations from enrolled bibliographic databas- 


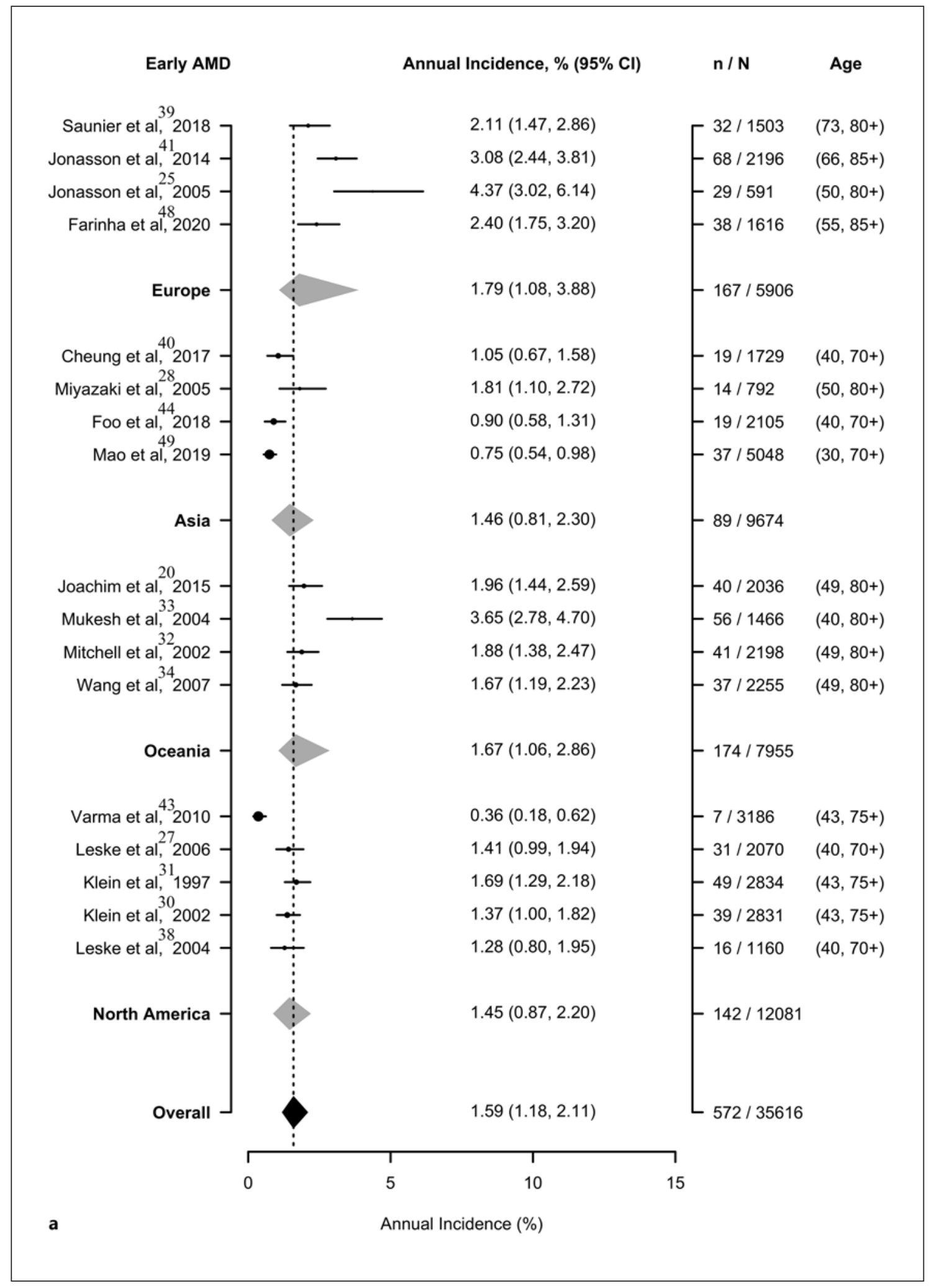

Fig. 2. a Forest plot for annual incidence of early AMD by region. AMD, age-related macular degeneration; CI, confidence interval. b Forest plot for annual incidence of late AMD by region. AMD, age-related macular degeneration; $\mathrm{CI}$, confidence interval.

(Figure continued on next page.) 


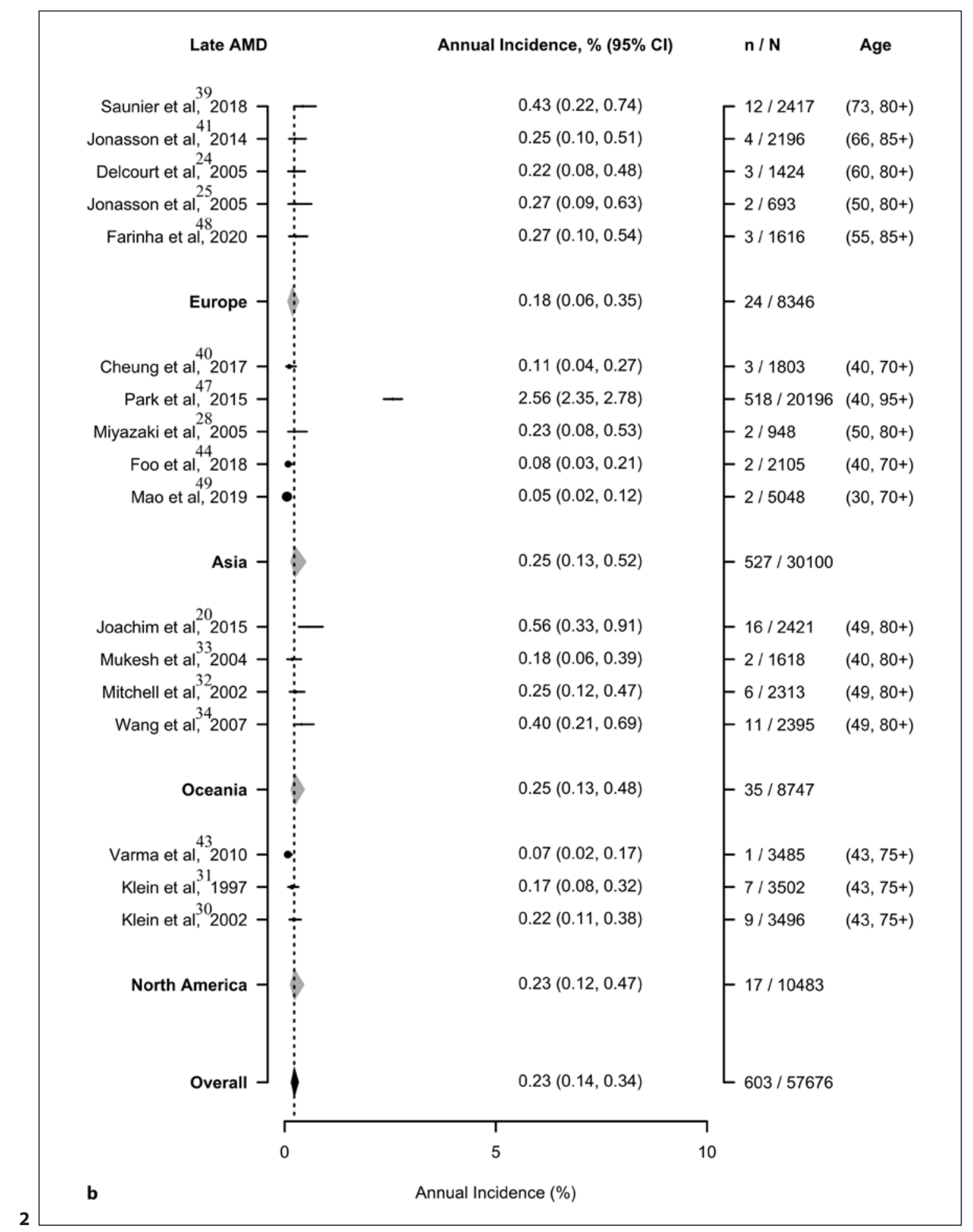

es. Finally, 30 studies involving 82,973 individuals from 12 countries and 5 continents were included in the current analysis. Among all included longitudinal population-based observational studies, 19 [20-38] were con- ducted before year 2000 and 11 [39-49] were conducted after year 2000. The age of the study participants ranged from 30 [35] to 98 [33] years old and follow-up duration ranged from 2 years [29] to 15.6 years [20]. Sample sizes 


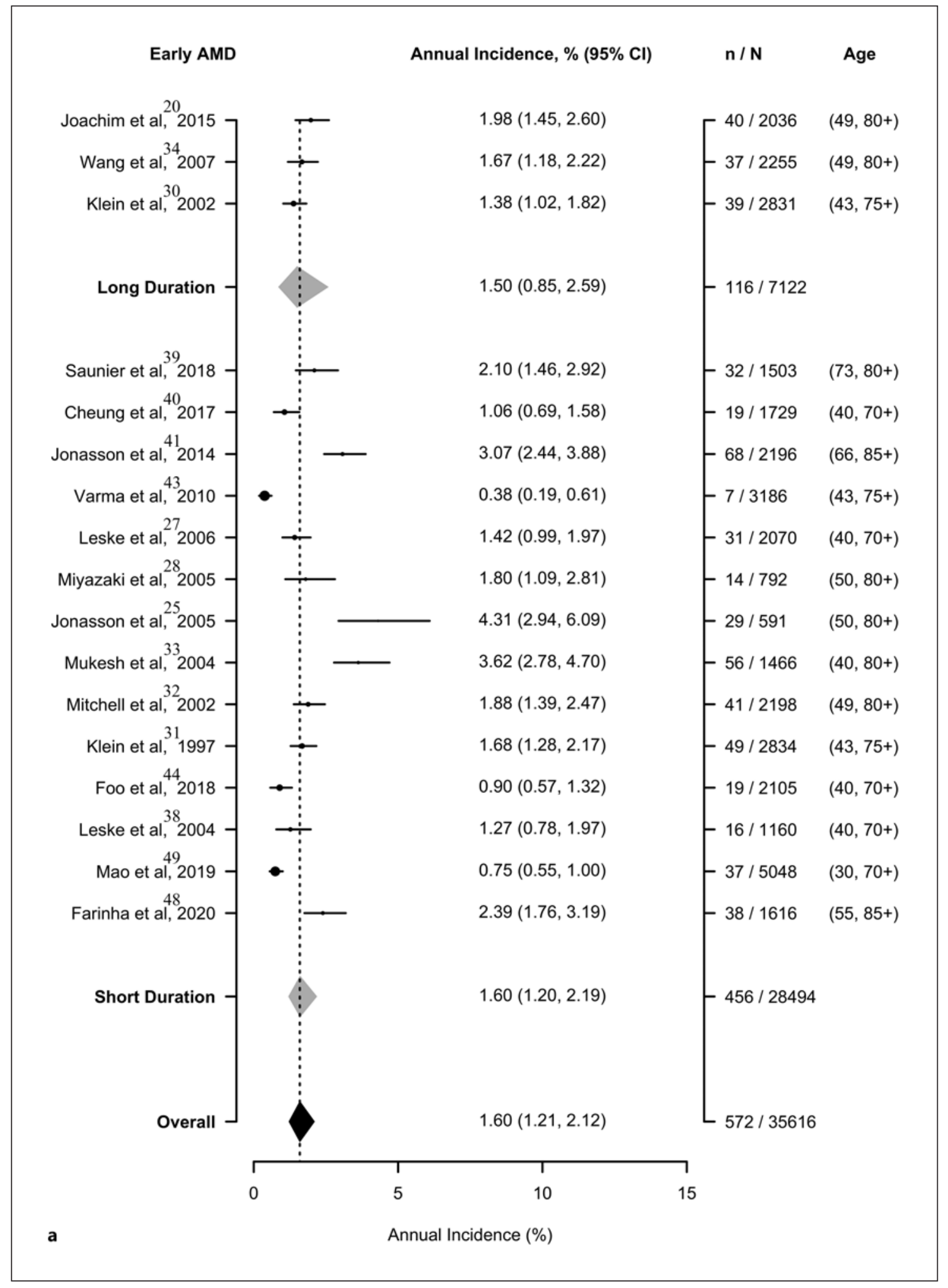

Fig. 3. a Forest plot for annual incidence of early AMD by observed duration. AMD, age-related macular degeneration; CI, confidence interval. b Forest plot for annual incidence of late AMD by observed duration. *Long duration: observed duration $\geq 10$ years; short duration: observed duration $<10$ years. AMD, age-related macular degeneration; CI, confidence interval.

(Figure continued on next page.)

Wang/Zhong/Zhang/Wu/Tham/Rim/ Kithinji/Wu/Cheng/Liang/Yu/Yang/Liu 


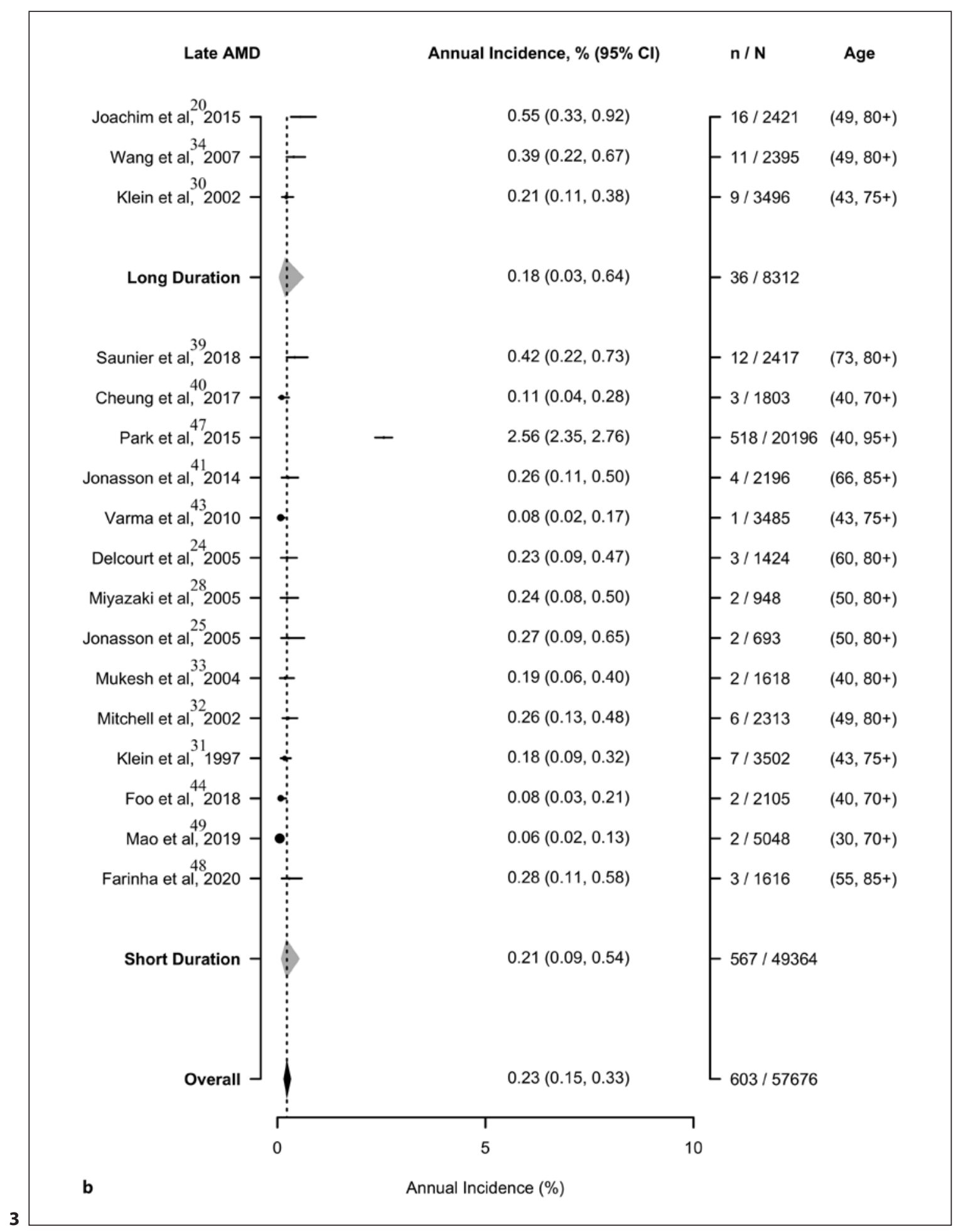

ranged from 313 in the Copenhagen City Eye Study/Denmark (CCES) $[22,23]$ to 20,196 in the Nationwide Population-Based Study (NPBS) [47], while response rates ranged from $50.1 \%$ in the Nakuru Eye Disease Cohort
Study (NEDCS) [45] to $97.3 \%$ in the CCES $[22,23]$. Overviews of studies regarding AMD incidence or progression are listed in online suppl. Table 1 and studies included for analysis are shown in online suppl. Table 2. 


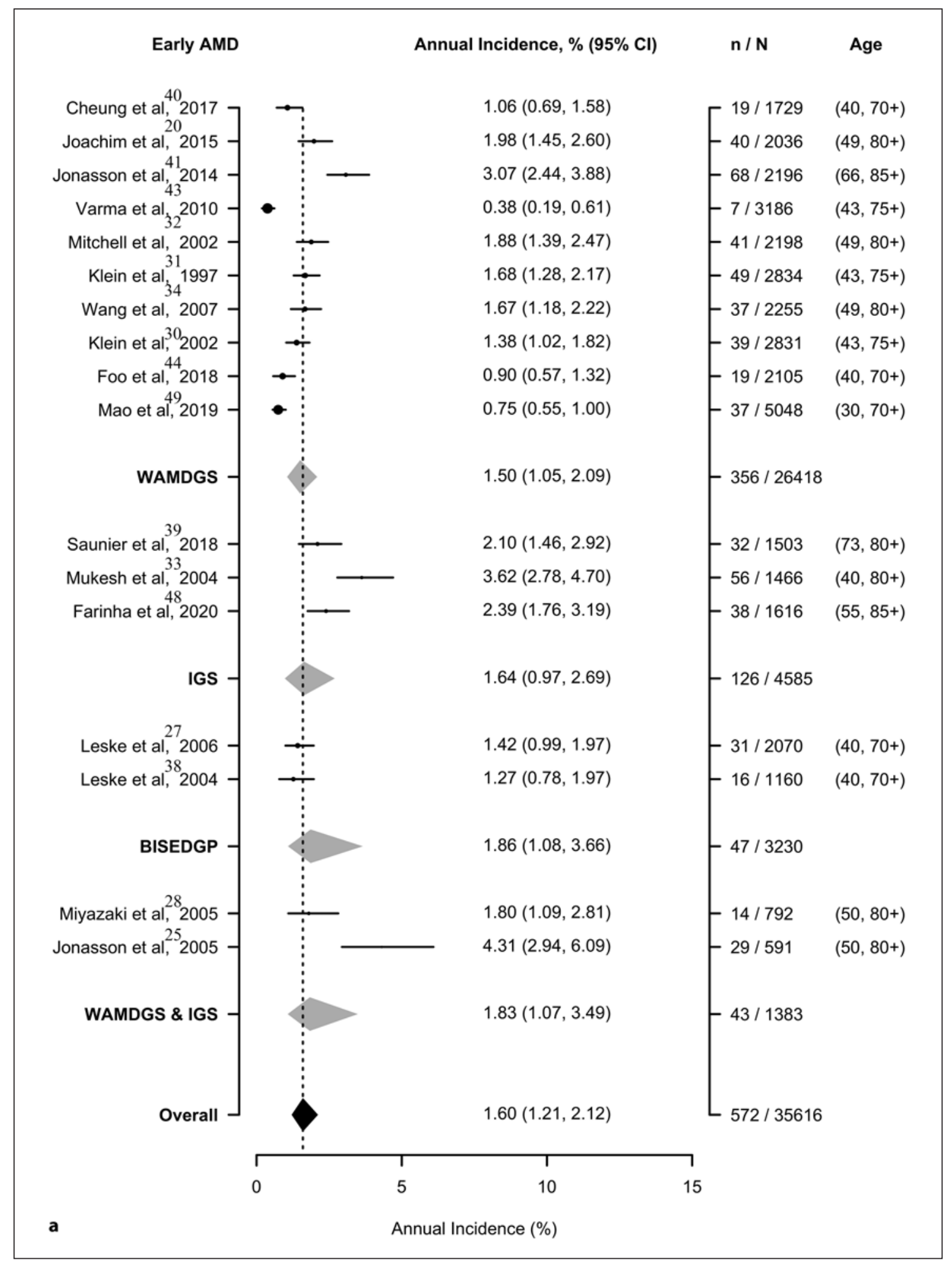

Fig. 4. a Forest plot for annual incidence of early AMD by AMD grading methods. AMD, age-related macular degeneration; CI, confidence interval; WAMDGS, Wisconsin Age-Related Macular Degeneration Grading System; IGS, international classification and grading system; BISEDGP, Barbados Eye Studies Grading
Protocol. $\mathbf{b}$ Forest plot for annual incidence of late AMD by AMD grading methods. AMD, age-related macular degeneration; CI, confidence interval; WAMDGS, Wisconsin Age-Related Macular Degeneration Grading System; IGS, international classification and grading system.

(Figure continued on next page.) 


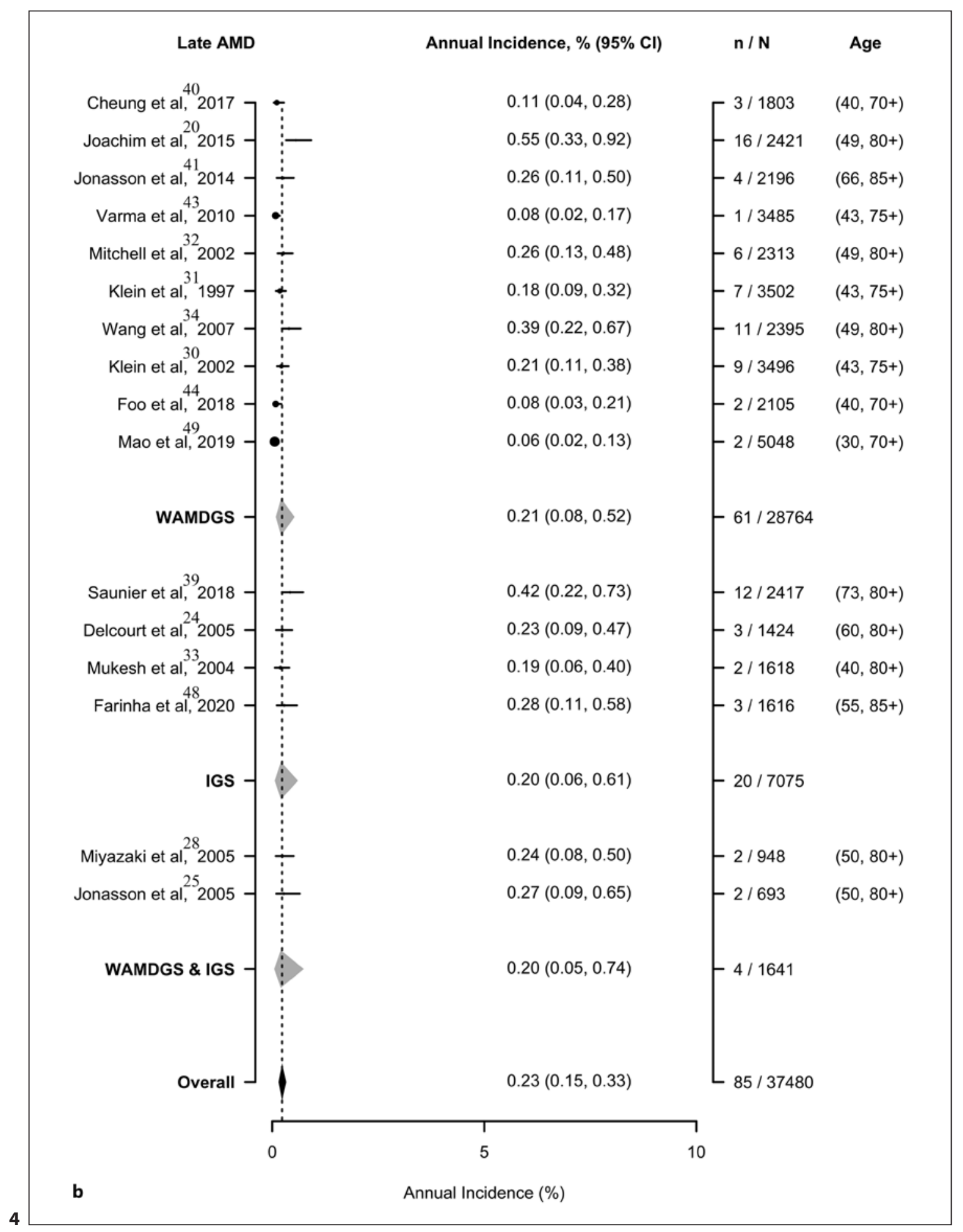

Subgroup Analysis by Region on AMD Incidence

Figure $2 \mathrm{a}, \mathrm{b}$ shows the annual incidence of early AMD and late AMD stratified by region, respectively. For early AMD, the overall annual incidence was 1.59 (95\% CI,
$1.18-2.11)$ per 100 person-years $\left(I^{2}=90.3 \%, p<0.0001\right)$. The annual incidence was 1.79 (95\% CrI, 1.08-3.88) per 100 person-years in Europe [25, 39, 41, 48], 1.46 (95\% CrI, 0.81-2.30) per 100 person-years in Asia [28, 40, 44, 


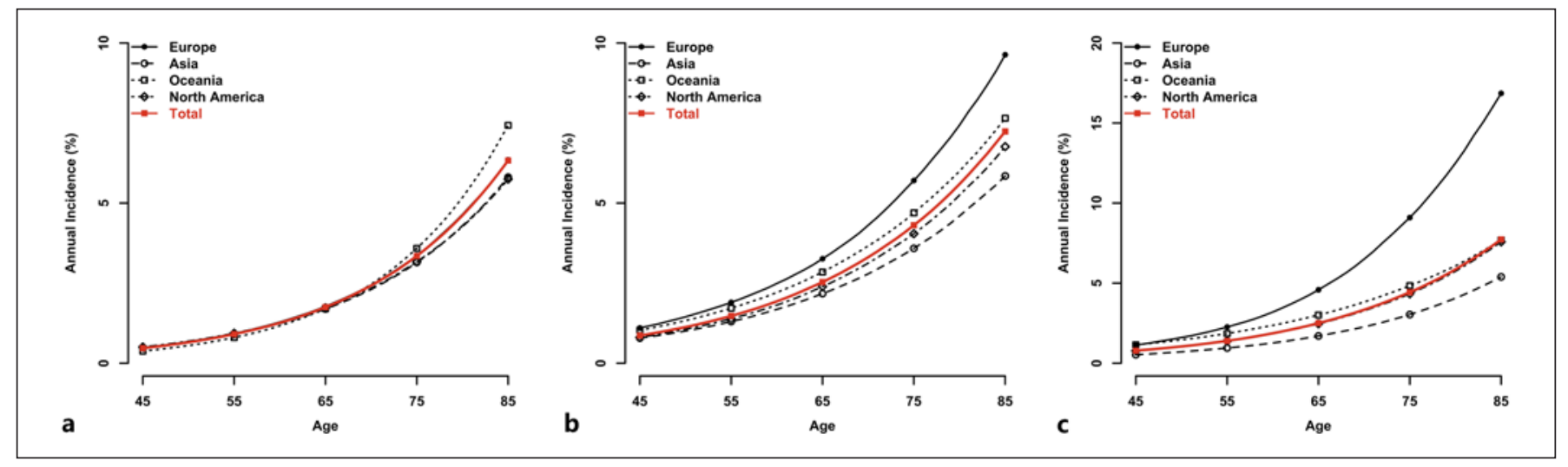

Fig. 5. Age trend of annual incidence of early AMD by country. a Overall age trend of annual incidence of early AMD by country. AMD, age-related macular degeneration. b Male-specific age trend of annual incidence of early AMD by country. AMD, age-related macular degeneration. $\mathbf{c}$ Female-specific age trend of annual incidence of early AMD by country. AMD, age-related macular degeneration

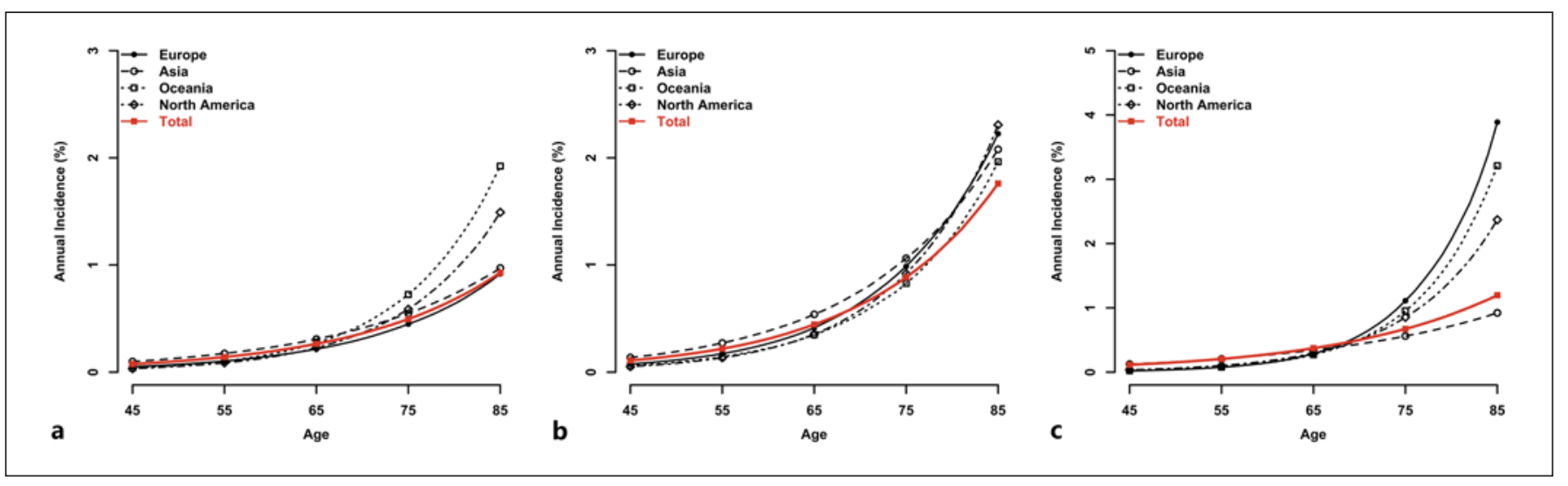

Fig. 6. Age trend of annual incidence of late AMD by country. a Overall age trend of annual incidence of late AMD by country. AMD, age-related macular degeneration. $\mathbf{b}$ Male-specific age trend of annual incidence of late AMD by country. AMD, age-related macular degeneration. c Female-specific age trend of annual incidence of late AMD by country. AMD, age-related macular degeneration.

49], 1.67 (95\% CrI, 1.06-2.86) per 100 person-years in Oceania [20, 32-34], and 1.45 (95\% CrI, 0.87-2.20) per 100 person-years in North America [27, 30, 31, 38, 43], where Europe had the highest annual incidence. For late AMD, the overall annual incidence was 0.23 (95\% CrI, $0.14-0.34)$ per 100 person-years $\left(I^{2}=95.7 \%, p<0.0001\right)$. The annual incidence was 0.18 (95\% CrI, $0.06-0.35$ ) per 100 person-years in Europe [24, 25, 39, 41, 48], 0.25 (95\% CrI, 0.13-0.52) per 100 person-years in Asia [28, 40, 44, 47, 49], 0.25 (95\% CrI, 0.130 .48 ) per 100 personyears in Oceania [20, 32-34] and 0.23 (95\% CrI, 0.12 $0.47)$ per 100 person-years in North America [30, 31, 43], where Asia and Oceania had the highest annual incidence.

\section{Subgroup Analysis by Observed Duration on AMD Incidence}

When classified by duration of observation, 6 studies $[20,22,23,26,30,34]$ were in the long duration group $(\geq 10$ years), and 24 studies [21, 24, 25, 27-29, 31-33, 35-49] were in the short duration group ( $<10$ years). Of the 30 included studies, 9 had a 5-year observation period [ $25,28,31-33,35$, $37,41,42]$. Annual incidences in studies with long duration were lower for both early AMD and late AMD (Fig. 3a, b).

\section{Subgroup Analysis by AMD Grading Methods on}

AMD Incidence

Of the total number of included studies, 14 graded AMD using the WAMDGS [20, 22, 23, 26, 30-32, 34, 37, 
Table 1. Projection of number of people (aged 45-85, in millions) who will have developed early AMD by regions

\begin{tabular}{llll}
\hline Region & 2030 & 2040 & 2050 \\
\hline Europe & $5.97(3.72,12.64)$ & $6.06(3.77,12.83)$ & $5.73(3.56,12.12)$ \\
Asia & $23.93(13.95,37.01)$ & $28.23(16.46,43.66)$ & $30.44(17.75,47.09)$ \\
Oceania & $0.26(0.17,0.45)$ & $0.30(0.20,0.51)$ & $0.34(0.22,0.56)$ \\
Northern America & $2.28(1.41,3.39)$ & $2.45(1.52,3.64)$ & $2.55(1.58,3.80)$ \\
World & $32.44(19.25,53.48)$ & $37.04(21.95,60.64)$ & $39.05(23.12,63.57)$ \\
\hline
\end{tabular}

AMD, age-related macular degeneration.

\begin{tabular}{llll}
\hline Region & 2030 & 2040 & 2050 \\
\hline Europe & $0.65(0.23,1.21)$ & $0.66(0.23,1.23)$ & $0.62(0.22,1.16)$ \\
Asia & $4.17(2.29,8.73)$ & $4.92(2.70,10.30)$ & $5.31(2.91,11.11)$ \\
Oceania & $0.04(0.02,0.08)$ & $0.05(0.03,0.09)$ & $0.05(0.03,0.10)$ \\
Northern America & $0.38(0.19,0.76)$ & $0.40(0.21,0.81)$ & $0.42(0.22,0.85)$ \\
World & $5.24(2.73,10.78)$ & $6.04(3.16,12.44)$ & $6.41(3.37,13.22)$ \\
\hline
\end{tabular}

AMD, age-related macular degeneration.
Table 2. Projection of number of people (aged 45-85, in millions) who will have developed late AMD by regions
$40,41,43,44,49]$. For early AMD, the annual incidence was highest when using BISEDGP [27, 38], with an overall incidence of $1.86 \%$ (95\% CrI, 1.08-3.66) per 100 person-years, followed by 1.83 (95\% CrI 1.07-3.49) when using WAMDGS \& IGS $[25,28], 1.64$ (95\% CrI, 0.97-2.69) when using IGS $[33,39,48]$, and 1.50 (95\% CrI, 1.05-2.09) when using WAMDGS [20, 30-32, 34, 40, 41, 43, 44, 49]. For late AMD, the annual incidence was 0.20 (95\% CrI, 0.05-0.74) when using WAMDGS \& IGS $[25,28], 0.21$ (95\% CrI, 0.08-0.52) per 100 person-years when using WAMDGS [20,30-32, 34, 40,41,43,44] and 0.20 per 100 person-years when using IGS $[24,33,39]$ on its own $(95 \%$ CrI, 0.06-0.61) (Fig. 4a, b).

Forest plots for annual incidence of early and late AMD by response rate, baseline year, and sample size are provided in the online suppl. Figures $1 \mathrm{a}, \mathrm{b}, 2 \mathrm{a}, \mathrm{b}$, and $3 \mathrm{a}$, b. Analysis of annual incidence on country after adjusted by age and gender are shown in the online suppl. Table 3.

Age Trend of Annual Incidence of Late AMD by Regions

As the figure shows, the annual incidence of AMD increased with age. Compared with the total trend, the incidence of early AMD in Oceania, followed by Europe, was above the overall incidence when age was over 75 years old (Fig. 5a). For either males (Fig. 5b) or females (Fig. 5c), the incidence of early AMD increased most rapidly in Europe after age 75. In the age trend of annual incidence of late AMD, Oceania had the highest incidence while Europe had the lowest incidence. Compar- ing with early AMD, the incidence of late AMD changed less (Fig. 6a). For males, incidence in Asia was the highest in most age groups (Fig. 6b), while the incidence was higher and increased most rapidly in Europe after 75 years of age for females (Fig. 6c). Besides, age trend of annual incidences of early and late AMD by country and sex are also presented in the online suppl., in which there is a difference between male and female (online suppl. Fig. $4 \mathrm{a}-\mathrm{f})$.

Projection Estimates of AMD for 2020-2050 by Regions

Tables 1 and 2 show the estimated number of people who will develop early or late AMD from 2030 to 2050 in decades stratified by regions. For the year 2030, the global projected new cases of early AMD would be 32.44 million (95\% CrI, 19.25-53.48), rising to 39.05 million (95\% CrI, 23.12-63.57) by 2050, with the largest number of people in Asia (30.44 million by 2050). Europe is expected to be second to Asia for early AMD (5.73 million by 2050), followed by Northern America (2.55 million), and then Oceania (0.34 million). For late AMD, the incidence is much lower than that of early AMD, with the same ranking of regions. In the year 2020, the global projected new cases for late AMD would be 5.24 million (95\% CrI 2.73-10.78), rising to 6.41 million (95\% CrI 3.37-13.22) by 2050 , with the largest number of people in Asia (5.31 million by 2050), followed by Europe (0.62 million), Northern America (0.42 million), and Oceania (0.05 million). 
Table 3. Risk factors for global incidence of early AMD

\begin{tabular}{lllr}
\hline Factor & Studies, $n$ & OR $(95 \%$ Crl $)$ & $p$ value \\
\hline Age & 6 & $1.09(1.05-1.13)$ & $<0.001$ \\
Female gender & 5 & $0.99(0.63-1.56)$ & 0.975 \\
Smoking & 7 & $1.59(1.17-2.17)$ & 0.003 \\
Alcohol consumption & 2 & $1.92(1.04-3.57)$ & 0.038 \\
Diabetes mellitus & 3 & $1.13(0.80-1.60)$ & 0.484 \\
Hypertension & 2 & $0.89(0.72-1.10)$ & 0.286 \\
CVDs & 2 & $1.21(0.69-2.12)$ & 0.499 \\
Pulse pressure & 2 & $1.00(0.99-1.01)$ & 0.999 \\
HDL-C & 2 & $1.35(1.01-1.81)$ & 0.040 \\
DBP & 2 & $1.02(1.00-1.04)$ & 0.052 \\
\hline
\end{tabular}

CVDs, cardiovascular diseases; HDL-C, high-density lipoprotein cholesterol; DBP, diastolic blood pressure; OR, odds ratio; Crls, credible intervals; AMD, age-related macular degeneration.

\section{Progression Rate on AMD}

The annual progression rate of AMD was 5.5 (95\% CrI: 2.3-8.8) per 100 person-years. Studies that have been included in the progression rate analysis were the Age and Gene/Environment Susceptibility Study (AGES) [41], the Barbados Eye Studies II (BISED II) [27], the Los Angeles Latino Eye Study (LALES) [43], and the Rotterdam Study (RTES) [29].

\section{Risk Factors for Occurrence of Early and Late AMD}

We summarized variables related to AMD incidence that were reported in the included studies. After pooling these factors, we found that age (OR: 1.09; 95\% CrI: 1.051.13) $[20,21,28,40-42]$, high-density lipoprotein cholesterol (HDL-C) $(1.35$; 95\% CrI: 1.01-1.81) [39, 41], smoking (1.59; 95\% CrI: 1.17-2.17) [20, 21,27, 28, 37, 40, 41], and alcohol consumption (1.92; 95\% CrI: 1.04-3.57) [37, $44]$ could be considered as pooled risk factors of early AMD incidence. For late AMD, only smoking [20, 27, 37] was investigated among 3 studies, and its risk toward late AMD incidence was 2.32 (95\% CrI: 1.04-5.18). A relative table of risk factors is shown in Tables 3 and 4.

\section{Discussion}

\section{Principle Findings}

To our knowledge, this is the first meta-analysis to summarize the global incidence rates and risk factors for both early and late AMD. In addition, we investigated progression rates of AMD among population-based longitudinal cohorts. In this systematic review and metaanalysis of 30 longitudinal population-based observa-
Table 4. Risk factors for global incidence of late AMD

\begin{tabular}{llll}
\hline Factor & Studies, $n$ & OR $(95 \% \mathrm{Crl})$ & $p$ value \\
\hline Smoking & 3 & $2.32(1.04-5.18)$ & 0.040 \\
\hline
\end{tabular}

AMD, age-related macular degeneration; OR, odds ratio; Crls, credible intervals.

tional studies [20-47], the main findings aimed to estimate the global incidence and progression of AMD and were as follows: First, the global annual incidence of AMD was different when stratified by different subgroups including regions, countries, observation durations, AMD grading methods, response rates, baseline years, and sample size. Second, in the year 2020, the estimated global new cases would be 32.44 million for early AMD and 5.24 million for late AMD, rising to 39.05 million and 6.41 million by 2050 , respectively. Third, the global annual incidence of both early and late AMD increased with age. Fourth, smoking was considered to be a risk factor for both early and late AMD, while age, HDL-C, and alcohol consumption were risk factors for early AMD only.

\section{Meaning of the Study and Comparison with Other Studies}

This meta-analysis included only 1 study from Africa [45] to examine cumulative incidence of AMD which might be caused by low income and limited infrastructure $[45,50]$. Since AMD is more prevalent among whites than blacks [51] and developed countries [10], more studies were conducted in Europe, North America, and Australia. Moreover, as presented in the Supplement, the annual incidence of early and late AMD increased with age, in which trends in females were more obvious than males. As Tables 1 and 2 shows, globally, the incidence of AMD is increasing in decades, with $>75 \%$ of new cases in Asia by 2050. Compared with Europe, the annual incidence of early AMD is lower in Asia. However, Asia has a large population base, and hence we will see the largest projected number of people with AMD. Besides, incidence of AMD in Asia is expected to increase more rapidly than other regions over the years [52], as age is a risk factor for $\mathrm{AMD}$ and the problem of the aging population in Asia is serious while the incidence in Europe being flat in the last 40 years according to our projection. The above points suggest that we should focus on prevention and control of AMD in Asia.

Age [20, 21, 28, 40-42], HDL [39, 41], alcohol consumption $[37,44]$, and smoking $[20,21,27,28,37,40$, 
41] are risk factors after analysis of results. Many factors such as gender [20,21, 40,45,47], hypertension [27, 41], diabetes $[27,41,45]$, and cardiovascular disease $[27,44]$ are no longer considered risk factors as their ORs were inconsistent across studies. For example, being female was considered to be a risk factor in the AGES [41], the Blue Mountains Eye Study III (BMES III) [20], the Singapore Malay Eye Study (SIMES) [40], and the NEDCS [45], while being male was considered as a risk factor in the Hisayama Study (HS) [21]. Ethnicity might partly explain the difference. The relationship between smoking and late AMD could be explained by shared risk factors (e.g., hypertension) or by potential unmeasured confounders (e.g., physical status) [53]. Although only 10 studies are included, all of risk factors are relative to incidence which overall results have not been reported till now. For further analysis, future studies should be performed in other regions and population such as Africa and Africans to obtain a more comprehensive understanding of how incidence can provide insight to time of exposure to risk factors.

Results from this study also showed that the annual progression rate $[27,29,41,43]$ of AMD was 5.5 per 100 person-year with $95 \% \mathrm{CrI}$ from $2.3 \%$ to $8.8 \%$. As only 4 studies were included, the results of the stratified analysis were not very reliable. This implies that more research is needed to better understand progression and facilitate progression analysis.

The assessment of the quality of the studies was performed in order to provide additional evidence of the internal and external validity of the data. According to NOS (online suppl. Tables 4, 5a, b), 27 of 30 studies included in our analysis were judged to be of low risk of bias probably because of that large, population-based, cohort studies had the advantage over other study designs in which they eliminate any temporal or causal ambiguity as exposure is before the onset of disease [53, 54]. Furthermore, the result funnel plot indicated evidence of significant publication bias (online suppl. Fig. 5a, b).

\section{Strengths and Limitations of the Study}

Strength of current study is that it comprises geographically and chronologically dispersed epidemiological data on global AMD incidence and progression specifically derived from longitudinal cohort studies to date. We had strict inclusion criteria to ensure including studies focus on incidence or progression rather than prevalence. Furthermore, our meta-analysis quantified global AMD incidence and its risk factors, enabling us to provide a contemporary estimation of AMD epidemiology.
This study was not without limitations. There were limited prospective epidemiological data on AMD incidence for some large populations, including India and Russia and nations from the continents of Africa and South America. We included cohort studies published in English and Chinese databases. However, variations in AMD incidence reported in a different language may exist, which may not have been included in this analysis. Another notable limitation of such a meta-analysis would be the significant heterogeneity, and this may have led to an overestimation or underestimation of incidences. Finally, in all included studies, AMD were diagnosed based on photographs. In clinic, a consensus group has recently recommended a multimodal image approach, including optical coherence tomography features, so that it may have led to an underestimation of the actual disease incidence. Notably, it may be unlike that this limitation in the study design inherent to almost any population- or community-based study might be involving these devices on site investigation. Future studies may further explore this topic.

\section{Unanswered Questions and Future Research}

Based on this systematic review, we recommend further studies of developing countries such as those in Africa and South America in order to obtain precise estimates of either early or late AMD across all regions. This is especially needed for studies pertaining to late AMD. Additionally, to avoid heterogeneity and facilitate metaanalysis, future studies should consider following a standardized method of reporting, including variables such as age and sex.

\section{Conclusions}

In summary, this systematic review provided an overview of the global incidence, progression, and risk factors of AMD over the past few decades and projected estimates of the number of people who will develop the disease from 2020 to 2050 across several regions in the world. Country-specific data are needed because of the varying population compositions and socioeconomic levels across different countries and cultures. Continued growth of the aging population will result in increasing numbers of cases of visual impairment related to AMD. Slowing the incidence of $A M D$, along with controlling its risk factors and developing future therapies, is required to reduce potential visual impairment burdens. Overall, this review provides useful information for the conceptualization 
and development of major public health strategies, such as prevention programs for AMD based on risk factors analysis.

\section{Statement of Ethics}

An ethics statement is not applicable because this study is based exclusively on published literature.

\section{Conflict of Interest Statement}

The authors have no conflicts of interest to declare.

\section{Funding Sources}

This article is supported by the National Natural Science Foundation of China (No. 81300783), China Postdoctoral Science Foundation (No. 2019TQ0358; 2019M661162), LiaoNing Revitalization Talents Program (No. XLYC1807082), and Shenyang Young and Middle-aged Science and Technology Innovation Talent Support Program (Grant No. RC190146). The funders had no role in the design and conduct of the study; the collection, management, analysis, interpretation of the data; the preparation, review, or approval of the manuscript; or the decision to submit the manuscript for publication.

\section{Author Contributions}

The original idea for the research was developed by L.L. and L.Z. L.Z. conducted the analysis with input from Y.W and L.L. Y.Z., Y.W., and L.L. conducted the searches, study selection, quality assessments, and other data extraction. Q.W., Y.T., T.H.R., C.C., J.W., H.L., H.Y., X.Y., and D.M.K. wrote the manuscript. All authors interpreted the findings and contributed to critical revision of the manuscript. All authors had full access to the data in the study and can take responsibility for the integrity of the data and the accuracy of the data analysis. L.L. is the guarantor. The corresponding author attests that all listed authors meet authorship criteria and that no others meeting the criteria have been omitted.

\section{Data Availability Statement}

No additional data are available.

\section{References}

1 VanNewkirk MR, Nanjan MB, Wang JJ, Mitchell P, Taylor HR, McCarty CA. The prevalence of age-related maculopathy: the visual impairment project. Ophthalmology. 2000 Aug;107(8):1593-600.

2 Keel S, Xie J, Foreman J, van Wijngaarden P, Taylor HR, Dirani M. Prevalence of age-related macular degeneration in Australia: the Australian national eye health survey. JAMA Ophthalmol. 2017 Nov 1;135(11):1242-9.

3 Jin G, Zou M, Chen A, Zhang Y, Young CA, Wang SB, et al. Prevalence of age-related macular degeneration in Chinese populations worldwide: a systematic review and metaanalysis. Clin Exp Ophthalmol. 2019 Nov; 47(8):1019-27.

4 Kawasaki R, Yasuda M, Song SJ, Chen SJ, Jonas JB, Wang JJ, et al. The prevalence of agerelated macular degeneration in Asians: a systematic review and meta-analysis. Ophthalmology. 2010 May;117(5):921-7.

5 Rudnicka AR, Jarrar Z, Wormald R, Cook DG, Fletcher A, Owen CG. Age and gender variations in age-related macular degeneration prevalence in populations of European ancestry: a meta-analysis. Ophthalmology. 2012 Mar;119(3):571-80.

6 Wong WL, Su X, Li X, Cheung CM, Klein R, Cheng CY, et al. Global prevalence of age-related macular degeneration and disease burden projection for 2020 and 2040: a systematic review and meta-analysis. Lancet Glob Health. 2014;2(2):e106-16.
7 Klein R, Davis MD, Magli YL, Segal P, Klein $\mathrm{BE}$, Hubbard L. The Wisconsin age-related maculopathy grading system. Ophthalmology. 1991;98(7):1128-34.

8 Bird AC, Bressler NM, Bressler SB, Chisholm IH, Coscas G, Davis MD, et al. An international classification and grading system for age-related maculopathy and agerelated macular degeneration. The International ARM Epidemiological Study Group. Surv Ophthalmol. 1995 Mar-Apr;39(5): $367-74$

9 Rudnicka AR, Kapetanakis VV, Jarrar Z, Wathern AK, Wormald R, Fletcher AE, et al. Incidence of late-stage age-related macular degeneration in American whites: systematic review and meta-analysis. Am J Ophthalmol. 2015 Jul;160(1):85-93.e3.

10 Gheorghe A, Mahdi L, Musat O. AGE-related macular degeneration. Rom J Ophthalmol. 2015 Apr-Jun;59(2):74-7.

11 Schachat AP, Hyman L, Leske MC, Connell $\mathrm{AM}, \mathrm{Wu}$ SY. Features of age-related macular degeneration in a black population. The Barbados Eye Study Group. Arch Ophthalmol. 19601995 Jun;113(6):728-35.

12 Rotenstein LS, Ramos MA, Torre M, Segal JB, Peluso MJ, Guille C, et al. Prevalence of depression, depressive symptoms, and suicidal ideation among medical students: a systematic review and metaanalysis. JAMA. 2016 Dec 6;316(21):221436.
13 Stang A. Critical evaluation of the NewcastleOttawa scale for the assessment of the quality of nonrandomized studies in meta-analyses. Eur J Epidemiol. 2010 Sep;25(9):603-5.

14 Ikram MK, van Leeuwen R, Vingerling JR, Hofman A, de Jong PT. Retinal vessel diameters and the risk of incident age-related macular disease: the Rotterdam Study. Ophthalmology. 2005 Apr;112(4):548-52.

15 Seddon JM, Francis PJ, George S, Schultz DW, Rosner B, Klein ML. Association of CFH Y402H and LOC387715 A69S with progression of age-related macular degeneration. JAMA. 2007 Apr 25;297(16):1793800.

16 United Nations. World population prospects 2019- population division. [cited 2020 May 12]. Available from: https://population.un. org/wpp/Download/Standard/Population/.

17 Jeffreys $\mathrm{H}$. Theory of probability. 3rd ed. Oxford: Clarendon Press; 1961

18 Siristatidis C, Sergentanis TN, Kanavidis P, Trivella M, Sotiraki M, Mavromatis I, et al. Controlled ovarian hyperstimulation for IVF: impact on ovarian, endometrial and cervical cancer-a systematic review and meta-analysis. Hum Reprod Update. 2013 Mar-Apr 19(2):105-23.

19 Sabanayagam C, Banu R, Chee ML, Lee R, Wang YX, Tan G, et al. Incidence and progression of diabetic retinopathy: a systematic review. Lancet Diabetes Endocrinol. 2019 Feb;7(2):140-9. 
20 Joachim N, Mitchell P, Burlutsky G, Kifley A, Wang JJ. The incidence and progression of age-related macular degeneration over 15 years: the blue mountains eye study. Ophthalmology. 2015 Dec;122(12):2482-9.

21 Yasuda M, Kiyohara Y, Hata Y, Arakawa S, Yonemoto K, Doi Y, et al. Nine-year incidence and risk factors for age-related macular degeneration in a defined Japanese population the Hisayama study. Ophthalmology. 2009 Nov;116(11):2135-40.

22 Buch H, Nielsen NV, Vinding T, Jensen GB, Prause JU, la Cour M. 14-year incidence, progression, and visual morbidity of age-related maculopathy: the Copenhagen City eye study. Ophthalmology. 2005 May;112(5):787-98.

23 Buch H, Vinding T, la Cour M, Jensen GB, Prause JU, Nielsen NV. Risk factors for agerelated maculopathy in a 14-year follow-up study: the Copenhagen City eye study. Acta Ophthalmol Scand. 2005 Aug;83(4):409-18.

24 Delcourt C, Lacroux A, Carriere I, Group PS. The three-year incidence of age-related macular degeneration: the "Pathologies Oculaires Liees a l'Age" (POLA) prospective study. Am J Ophthalmol. 2005 Nov;140(5):924-6.

25 Jonasson F, Arnarsson A, Peto T, Sasaki H, Sasaki K, Bird AC. 5-year incidence of agerelated maculopathy in the Reykjavik eye study. Ophthalmology. 2005 Jan;112(1):1328.

26 Klein R, Klein BE, Knudtson MD, Meuer SM, Swift M, Gangnon RE. Fifteen-year cumulative incidence of age-related macular degeneration: the Beaver Dam eye study. Ophthalmology. $2007 \mathrm{Feb} ; 114(2): 253-62$.

27 Leske MC, Wu SY, Hennis A, Nemesure B, Yang L, Hyman L, et al. Nine-year incidence of age-related macular degeneration in the Barbados Eye Studies. Ophthalmology. 2006 Jan;113(1):29-35.

28 Miyazaki M, Kiyohara Y, Yoshida A, Iida M, Nose $\mathrm{Y}$, Ishibashi T. The 5-year incidence and risk factors for age-related maculopathy in a general Japanese population: the Hisayama study. Invest Ophthalmol Vis Sci. 2005 Jun; 46(6):1907-10

29 Klaver CC, Assink JJ, van Leeuwen R, Wolfs RC, Vingerling JR, Stijnen T, et al. Incidence and progression rates of age-related maculopathy: the Rotterdam study. Invest Ophthalmol Vis Sci. 2001 Sep;42(10):2237-41.

30 Klein R, Klein BE, Tomany SC, Meuer SM, Huang GH. Ten-year incidence and progression of age-related maculopathy: the Beaver Dam eye study. Ophthalmology. 2002 Oct; 109(10):1767-79.
31 Klein R, Klein BEK, Jensen SC, Meuer SM. The five-year incidence and progression of age-related maculopathy. Ophthalmology. 1997;104(1):7-21.

32 Mitchell P, Wang JJ, Foran S, Smith W. Fiveyear incidence of age-related maculopathy lesions: the Blue Mountains eye study. Ophthalmology. 2002 Jun;109(6):1092-7.

33 Mukesh BN, Dimitrov PN, Leikin S, Wang JJ, Mitchell P, McCarty CA, et al. Five-year incidence of age-related maculopathy: the visual impairment project. Ophthalmology. 2004 Jun;111(6):1176-82.

34 Wang JJ, Rochtchina E, Lee AJ, Chia EM, Smith W, Cumming RG, et al. Ten-year incidence and progression of age-related maculopathy: the blue Mountains eye study. Ophthalmology. 2007 Jan;114(1):92-8.

35 Bressler NM, Munoz B, Maguire MG, Vitale SE, Schein OD, Taylor HR, et al. Five-year incidence and disappearance of drusen and retinal pigment epithelial abnormalities. Waterman study. Arch Ophthalmol. 19601995 Mar; 113(3):301-8.

36 Chang MA, Bressler SB, Munoz B, West SK. Racial differences and other risk factors for incidence and progression of age-related macular degeneration: Salisbury Eye Evaluation (SEE) project. Invest Ophthalmol Vis Sci. 2008 Jun;49(6):2395-402.

37 Coleman AL, Seitzman RL, Cummings SR, Yu F, Cauley JA, Ensrud KE, et al. The association of smoking and alcohol use with agerelated macular degeneration in the oldest old: the study of osteoporotic fractures. Am J Ophthalmol. 2010 Jan;149(1):160-9.

38 Leske MC, Wu SY, Hyman L, Hennis A, Nemesure B, Schachat AP, et al. Four-year incidence of macular changes in the Barbados eye studies. Ophthalmology. 2004 Apr; 111(4):706-11.

39 Saunier V, Merle BMJ, Delyfer MN, Cougnard-Grégoire A, Rougier MB, Amouyel P, et al. Incidence of and risk factors associated with age-related macular degeneration: fouryear follow-up from the ALIENOR study. JAMA Ophthalmol. 2018 May 1;136(5):47381.

40 Cheung CMG, Ong PG, Neelam K, Tan PC, Shi Y, Mitchell P, et al. Six-year incidence of age-related macular degeneration in Asian Malays: the Singapore Malay eye study. Ophthalmology. 2017 Sep;124(9):1305-13.

41 Jonasson F, Fisher DE, Eiriksdottir G, Sigurdsson S, Klein R, Launer LJ, et al. Five-year incidence, progression, and risk factors for age-related macular degeneration: the age, gene/environment susceptibility study. Ophthalmology. 2014 Sep;121(9):1766-72.

42 You QS, Xu L, Yang H, Li YB, Wang S, Wang $J D$, et al. Five-year incidence of age-related macular degeneration: the Beijing eye study. Ophthalmology. 2012 Dec;119(12):2519-25.
43 Varma R, Foong AW, Lai MY, Choudhury F, Klein R, Azen SP, et al. Four-year incidence and progression of age-related macular degeneration: the Los Angeles Latino eye study. Am J Ophthalmol. 2010 May;149(5):741-51.

44 Foo VHX, Yanagi Y, Nguyen QD, Sabanayagam C, Lim SH, Neelam K, et al. Six-year incidence and risk factors of age-related macular degeneration in Singaporean Indians: the Singapore Indian eye study. Sci Rep. 2018 Jun 11;8(1):8869.

45 Bastawrous A, Mathenge W, Peto T, Shah N, Wing $\mathrm{K}$, Rono $\mathrm{H}$, et al. Six-year incidence and progression of age-related macular degeneration in Kenya: Nakuru eye disease cohort study. JAMA Ophthalmol. 2017 Jun 1;135(6): 631-8.

46 Chaker L, Buitendijk GH, Dehghan A, Medici M, Hofman A, Vingerling JR, et al. Thyroid function and age-related macular degeneration: a prospective population-based cohort study - the Rotterdam study. BMC Med. 2015 Apr 23;13:94.

47 Park SJ, Kwon KE, Choi NK, Park KH, Woo SJ. Prevalence and incidence of exudative agerelated macular degeneration in South Korea: a nationwide population-based study. Ophthalmology. 2015 Oct;122(10):2063-70.e1.

48 Farinha CVL, Cachulo ML, Alves D, Pires I, Marques JP, Barreto $P$, et al. Incidence of agerelated macular degeneration in the central region of Portugal: the Coimbra eye study-report 5. Ophthalmic Res. 2019;61(4):226-35.

49 Mao F, Yang X, Yang K, Cao X, Cao K, Hao J, et al. Six-year incidence and risk factors for age-related macular degeneration in a rural Chinese population: the Handan eye study. Invest Ophthalmol Vis Sci. 2019 Dec 2;60(15): 4966-71.

50 World Bank. GDP (current US. [cited 2020 May 12]. Available from: https://data.worldbank.org/indicator/NY.GDP.MKTP. $\mathrm{CD}$ ? end $=2018 \&$ name_desc $=$ true \&start $=$ 2018\&view=map.

51 Friedman DS, Katz J, Bressler NM, Rahmani B, Tielsch JM. Racial differences in the prevalence of age-related macular degeneration: the Baltimore eye survey. Ophthalmology. 1999;106(6):1049-55.

52 World Bank. Population ages 65 and above, total. [cited 2020 May 12]; Available from: https://data.worldbank.org/indicator/ SP.POP.65UP.TO? end $=2018 \&$ name desc $=f$ alse\&start $=1960 \&$ type $=$ shaded $\&$ view $=$ map.

53 Chakravarthy U, Wong TY, Fletcher A, Piault E, Evans C, Zlateva G, et al. Clinical risk factors for age-related macular degeneration: a systematic review and meta-analysis. BMC Ophthalmol. 2010;10(31):31.

54 Wong TY, Hyman L. Population-based studies in ophthalmology. Am J Ophthalmol. 2008 Nov;146(5):656-63. 